\title{
Diffusive transport properties in monovalent and divalent metal-ion halide melts: A computer simulation study
}

\author{
J. Trullàs \\ Departament de Física i Enginyeria Nuclear, Universitat Politècnica de Catalunya, Sor Eulàlia d'Anzizu, Campus Nord, Mòdul B4-B5, \\ 08034 Barcelona, Spain \\ J. A. Padró \\ Departament de Física Fonamental, Universitat de Barcelona, Diagonal, 647, 08028 Barcelona, Spain \\ (Received 5 August 1996; revised manuscript received 31 October 1996)
}

\begin{abstract}
Self- and cross-velocity correlation functions and related transport coefficients of molten salts are studied by molecular-dynamics simulation. Six representative systems are considered, i.e., $\mathrm{NaCl}$ and $\mathrm{KCl}$ alkali halides, $\mathrm{CuCl}$ and $\mathrm{CuBr}$ noble-metal halides, and $\mathrm{SrCl}_{2}$ and $\mathrm{ZnCl}_{2}$ divalent metal-ion halides. Computer simulation results are compared with experimental self-diffusion coefficients and electrical conductivities. Special attention is paid to dynamic cross correlations and their dependence on the Coulomb interactions as well as on the size and mass differences between anions and cations.[S0163-1829(97)13017-X]
\end{abstract}

\section{INTRODUCTION}

During the last years, computer simulation has proved to be a powerful tool for understanding the microscopic properties of liquids. Molecular-dynamics (MD) simulation is among the most suitable methods for investigating the dynamic properties. Molten alkali halides are relatively simple binary liquids with properties that may be realistically reproduced by simulations using same potential models as for the solid phases. ${ }^{1}$ These liquids were already studied at the early stages of computer simulation. ${ }^{2}$ Computer simulation studies of molten noble-metal halides are more recent. Stafford et $a l^{3}$ showed that potentials including a parameter which could be associated with an effective ionic charge $(|z|<1)$ allow us to reproduce the main structural trends of these systems. ${ }^{3}$ The available potentials for 2-1 melts are less satisfactory (this point will be discussed further on). To our knowledge there are no realistic potential models for more complex molten salts (trihalide melts, molten salt mixtures, ....). Microscopic properties of different classes of molten salts have been recently reviewed. ${ }^{4-6}$

Computer simulations of molten salts have been mainly devoted to the structural properties. Fewer simulations of the dynamic properties, ordinarily restricted to individual properties such as velocity autocorrelation functions and selfdiffusion coefficients, have been carried out. The study of collective properties such as electrical conductivities and distinct diffusion coefficients is more difficult because they depend on the correlations among the motions of the different particles in the system. To our knowledge, the only MD study on the dynamic cross correlations has been the one by Hansen and McDonald ${ }^{7,8}$ for a simple molten salt model where the only difference between anions and cations is the sign of the electric charge. Ciccotti, Jacucci, and McDonald ${ }^{9}$ have calculated the collective transport properties in molten alkali halides by nonequilibrium MD simulation but the effects of the cross correlations were not analyzed in this work.

Self- and distinct-velocity correlation functions and related diffusion coefficients have been suggested as basic pieces for the study of transport properties in multicomponent liquids ${ }^{10}$ (definitions of these properties are given in Sec. II). Velocity correlation functions play a fundamental role in statistical mechanics and both self- and distinctdiffusion coefficients may be obtained from experiment. ${ }^{11,12}$ Self-properties provide information on the motions of single atoms whereas distinct properties supply a measure of the coupling between the velocities of different particles. Distinct diffusion coefficients have been proposed to probe the ion-ion interaction models in electrolyte solutions. ${ }^{10}$ Experimental distinct diffusion coefficients for 14 binary nonelectrolyte mixtures at different concentrations have been reported recently. ${ }^{11}$ One of the reasons for the increasing interest on the distinct properties in multicomponent liquids may be that, unlike other transport properties, they can be related to the interactions between pairs of particles of both the same and different species. ${ }^{10,11}$

An important complication for the interpretation of distinct properties lies in the fact that, in principle, they are defined (or measured) with respect to some specific reference frame $R$ and their values are markedly dependent on the choice of $R .^{12,13}$ The mass-fixed (also called barycentric) and the number-fixed reference frames are commonly used in theoretical treatments. Results from ordinary MD simulations, at constant energy and moment, correspond to the mass-fixed reference frame. ${ }^{13}$ The solvent fixed is the most natural frame for the study of electrolyte solutions. Almost all diffusion measurements are carried out in the volumefixed frame. ${ }^{11,12}$ We performed a preliminary MD study of the distinct properties in molten salts. ${ }^{14}$ However, the results of this study could not be suitably interpreted because the influence of the reference frame was predominant. Recently, we proposed a set of self- and distinct-time correlation functions and corresponding diffusion coefficients which are independent of the reference frame. ${ }^{15}$ These functions were already applied to the study of binary mixtures of soft spheres ${ }^{16}$ and are the ones used in this work.

The aim of this paper is to analyze the diffusive transport properties of several molten salts by MD simulation, paying 
special attention to the distinct properties and electrical conductivities. Six ionic binary mixtures, as representative of three different molten salts classes, have been considered. Monohalide melts may be divided into molten alkali halides and noble-metal halides. ${ }^{6}$ The microscopic properties of the former, which are frequently taken as prototypical of ionic liquids, are strongly affected by charge ordering. ${ }^{17}$ In the case of the noble-metal halides, which show superionic solid phases, ${ }^{18}$ the microscopic properties are very sensitive to the size differences between anions and cations. ${ }^{17} \mathrm{KCl}$ and $\mathrm{NaCl}$ have been chosen as representative of alkali halides and $\mathrm{CuCl}$ and $\mathrm{CuBr}$ as representative of noble-metal halides. In $\mathrm{CuCl}$, the bigger ion $\left(\mathrm{Cl}^{-}\right)$is the lighter whereas in $\mathrm{CuBr}$ the bigger ion $\left(\mathrm{Br}^{-}\right)$is the heavier. $\mathrm{SrCl}_{2}$ and $\mathrm{ZnCl}_{2}$ have been chosen as representative of the 2-1 molten salts. The former melts at high temperature from a fluorite-type superionic conductor, whereas the latter melts at lower temperature and its ionic conductivity is markedly smaller. ${ }^{6}$

The MD simulations of this paper have been carried out by assuming the most usual pairwise potential models in the literature. The reliability of these models was ordinarily checked by considering only equilibrium properties. We have extended the test to the transport properties, namely self-diffusion coefficients and electrical conductivities. The properties of simulated and actual systems differ significantly for the 2-1 melts and MD simulations in this work would be merely considered as simple models of this type of molten salts.

\section{TIME CORRELATION FUNCTIONS AND TRANSPORT PROPERTIES: DEFINITIONS}

The properties considered in this work have been calculated according to the definitions given in previous papers. ${ }^{15,16}$ In a binary system, we define the relative velocity correlation function $\left[\Lambda_{12}(t)\right]$ as the time correlation function of the relative velocity of the center of mass of species 1 with respect to the center of mass of species 2 (Ref. 16)

$$
\Lambda_{12}(t) \equiv \frac{1}{3} x_{1} x_{2}\left\{N\left\langle\left[u_{1}(t)-u_{2}(t)\right]\left[u_{1}(0)-u_{2}(0)\right]\right\rangle\right\}_{\infty},
$$

where $\{\cdots\}_{\infty}$ indicates the thermodynamic limit operation, $\langle\cdots\rangle$ denotes the average over the canonical ensemble, $x_{\alpha}$ $=N_{\alpha} / N$ is the ionic fraction, and $u_{\alpha}(t)$ is the velocity of the center of mass of particles of species $\alpha$

$$
u_{\alpha}(t) \equiv N_{\alpha}^{-1} \sum_{i=1}^{N_{\alpha}} u_{i}(t) \quad(\alpha=1,2),
$$

where $N_{\alpha}$ is the number of particles of species $\alpha$, and $u_{i}(t)$ is the velocity of the particle $i$ of species $\alpha$ on any reference frame $R$.

$\Lambda_{12}(t)$ is a time correlation function which is independent of $R$ and may be easily calculated in MD simulations. Moreover, this function is a combination of the self- and crossvelocity correlation functions. Thus, it can be resolved into self $\left[\Lambda_{12}^{0}(t)\right]$ and distinct $\left[\Lambda_{12}^{d}(t)\right]$ contributions as follows: ${ }^{6}$

$$
\Lambda_{12}(t)=\Lambda_{12}^{0}(t)+x_{1} x_{2} \Lambda_{12}^{d}(t) .
$$

The contribution of the self-velocity correlations is

$$
\Lambda_{12}^{0}(t)=x_{2} \Lambda_{1}^{s}(t)+x_{1} \Lambda_{2}^{s}(t) .
$$

$\Lambda_{1}^{s}(t)$ and $\Lambda_{2}^{s}(t)$ are the well-known velocity autocorrelation functions,

$$
\Lambda_{\alpha}^{s}(t)=\frac{1}{3}\left\{\left\langle u_{i}(t) u_{i}(0)\right\rangle\right\}_{\infty} \quad(\alpha=1,2) .
$$

The contribution of the distinct velocity correlations may be written ${ }^{16}$

$$
\Lambda_{12}^{d}(t)=\Lambda_{11}^{d R}(t)+\Lambda_{22}^{d R}(t)-2 \Lambda_{12}^{d R}(t),
$$

where the right side terms are the time correlation functions among the velocities of distinct particles of the same $\left[\Lambda_{11}^{d R}(t), \Lambda_{22}^{d R}(t)\right]$ or different $\left[\Lambda_{12}^{d R}(t)\right]$ species $^{13,15,16}$

$$
\Lambda_{\alpha \beta}^{d R}(t)=\frac{1}{3}\left\{N\left\langle u_{i}(t) u_{j}(0)\right\rangle\right\}_{\infty} \quad(\alpha, \beta=1,2),
$$

where $i$ is a particle of species $\alpha$ and $j$ is a particle of species $\beta$.

The $\Lambda_{1}^{s}(t)$ functions are independent of $R$ whereas the $\Lambda_{\alpha \beta}^{d R}(t)$ functions, which are those considered in earlier MD studies, ${ }^{13}$ are markedly dependent on $R .{ }^{13}$ We emphasize that $\Lambda_{12}^{d}(t)$ is independent of $R$, despite being a combination of $\Lambda_{\alpha \beta}^{d R}(t)$ functions. This independence is a useful property which may be easily deduced from Eq. (3) by taking into account that both $\Lambda_{12}(t)$ and $\Lambda_{12}^{0}(t)$ are independent of $R$. The independence of $R$ of $\Lambda_{12}^{d}(t)$ was also observed by Mills et al. ${ }^{11}$ by manipulating the experimental distinct diffusion coefficients in different liquid mixtures.

The initial values $\Lambda(0)$ do not provide significant information since they are simply dependent on the temperature and the mass of particles. ${ }^{16} \mathrm{We}$ will represent the time correlation functions in the normalized form

$$
C(t)=\Lambda(t) /|\Lambda(0)| .
$$

A useful measure of the cross correlations effects is given by $\delta_{12}(t)$

$$
\delta_{12}(t)=x_{1} x_{2} \Lambda_{12}^{d}(t) / \Lambda_{12}^{0}(0) .
$$

Taking into account Eq. (3) and that $\Lambda_{12}(0)=\Lambda_{12}^{0}(0)$, we obtain

$$
C_{12}(t)=C_{12}^{0}(t)+\delta_{12}(t) .
$$

It should be noted that $\Lambda_{12}^{d}(t)$ and $\delta_{12}(t)$ also reflect the differences between the species in the system. So, for "mixtures" of two identical species $\Lambda_{12}^{d}(t)=\delta_{12}(t)=0$ [see Eq. (6)].

The diffusion coefficients $D_{12}, D_{12}^{0}, D_{1}^{s}, D_{2}^{s}$, and $D_{12}^{d}$ are defined as the time integrals of the corresponding velocity correlation functions

$$
D=\int_{0}^{\infty} \Lambda(t) d t .
$$

Diffusion coefficients may be alternatively calculated using Einstein-like relations for the displacement correlation functions. ${ }^{15}$ The relations among different diffusion coefficients are analogous to those among the corresponding time correlations functions [Eqs. (3), (4), (6), and (9)]. It is useful to define a dimensionless coefficient 


$$
\delta_{12}=x_{1} x_{2} \frac{D_{12}^{d}}{D_{12}^{0}} .
$$

By integration of Eq. (10) and taking into account Eqs. (8) and (11), we obtain

$$
D_{12}=D_{12}^{0}\left(1+\delta_{12}\right) .
$$

The systems considered in this work are mixtures of positive and negative ions. For the sake of clarity species 1 will be denoted as + and species 2 as - In these mixtures, the electric conductivity is related to the interdiffusion coefficient by the equation ${ }^{15}$

$$
\sigma=e^{2} \beta \frac{N}{V} z_{+} z_{-} D_{+-},
$$

where $e$ is the proton charge, $\beta=\left(k_{B} T\right)^{-1}$, and $z_{\alpha}$ is the signed charge number of the species $\alpha$. In order to analyze the contributions of the self- and distinct correlations to $\sigma$, we can write ${ }^{15}$

$$
\sigma=\sigma^{0}\left(1+\delta_{+-}\right),
$$

where $\sigma^{0}$ is the self-contribution

$$
\sigma^{0}=e^{2} \beta \frac{N}{V}\left(x_{+} z_{+}^{2} D_{+}^{s}+x_{-} z_{-}^{2} D_{-}^{s}\right) .
$$

Equation (16) is called the Nernst-Einstein relation. The deviations of this relation are frequently measured in terms of $\Delta \equiv-\delta_{+-} .8$

\section{RESULTS AND DISCUSSION}

MD simulations of systems made up of 108 anions and 108 cations in the case of monohalide melts and 216 anions and 108 cations in the case of dihalide melts have been carried out. Periodic boundary conditions have been considered and the long-ranged Coulomb interactions have been calculated according to the Ewald method. Temperatures, densities, and interionic potentials for each simulated system will be detailed further on. The $\Lambda_{+}^{s}(t), \Lambda_{-}^{s}(t)$, and $\Lambda_{+-}(t)$ correlation functions have been calculated from the ionic velocities during the MD simulations and other properties have been determined from them according to the equations in Sec. II. The diffusion coefficients have been also calculated from the corresponding displacement correlation functions. ${ }^{15}$ The agreement between the results obtained with the two methods is satisfactory. We have performed runs of about 1500 ps with a time step of $5 \times 10^{-3}$ ps.

\section{A. Monohalide melts}

\section{Alkali halides: $\mathrm{KCl}, \mathrm{NaCl}$}

MD simulations of molten $\mathrm{KCl}$ at two temperatures ( $T$ $=1173$ and $1273 \mathrm{~K}$ ) and molten $\mathrm{NaCl}$ at three temperatures ( $T=1073,1173$, and $1273 \mathrm{~K}$ ) have been carried out. For the sake of brevity only the results at one temperature are reported in this paper (Fig. 1 and Table I) but results at different temperatures have been taken into account in the discussion and are available on request. The effective pair interionic potentials of Born-Mayer-Huggins in the form and parametrization given by Fumi and Tosi ${ }^{19}$ and reviewed by Sangster and Dixon ${ }^{1}$ have been used. More details and a plot of the potentials for $\mathrm{NaCl}$ are shown in Ref. 17. Despite their simplicity these potential models account for the main structural features of molten alkali halides and are widely used in both simulations and theoretical calculations. ${ }^{4,6}$ The MD structure of $\mathrm{NaCl}$ is in accordance with diffraction experiments ${ }^{20}$ and results are not significantly improved by considering polarizable ions. ${ }^{21}$

Changes with temperature of $D_{\alpha}^{s}$ and $\sigma$ obtained from MD are consistent with those from experiment. As in other studies, ${ }^{9}$ the $D_{-}^{s}$ results from MD are in agreement with experiment whereas the $D_{+}^{s}$ values from MD are too low. However, it should be pointed out that the experimental data obtained by different authors show large discrepancies (see, for example, $D^{s}$ for $\mathrm{NaCl}$ in Refs. 22 and 23). Jacucci, McDonald, and Rahman ${ }^{24}$ observed that the $D_{\alpha}^{s}$ values, and especially $D_{+}^{s}$, increase when polarization effects are considered. Both $D_{+}^{s}$ and $D_{-}^{s}$ coefficients resulting from recent MD simulations using a polarizable model ${ }^{21}$ are markedly higher than the experimental data. MD and experimental results for $\sigma$ are in good agreement. However the value of $\sigma$ is almost entirely determined by the ionic masses and diameters ${ }^{9}$ and it is rather insensitive to the ionic polarizability. ${ }^{24}$

More detailed information on the ionic motions at short time scales is provided by the $C_{\alpha}^{s}(t)$ functions (Fig. 1). These functions show negative values (backscattering) which reflect a high probability of large-angle deflections in the "collisions" of ions with their neighbors. $C_{+}^{s}(t)$ and $C_{-}^{s}(t)$ for $\mathrm{KCl}$ are very close (and also $D_{+}^{s} \cong D_{-}^{s}$ ) but noticeable differences may be observed for $\mathrm{NaCl}$. As with Lennard-Jones mixtures, ${ }^{25}$ backscattering is more pronounced for lighter ions. The oscillations of $C_{\alpha}^{s}(t)$ are associated with "rattling" motions experienced by ions in the temporary cage formed by their neighboring unlike ions ${ }^{26}$ (the structure of these systems is characterized by a regular alternation of concentric shells of oppositely charged ions).

A quantitative measure of the effects of the distinct velocity correlations is given by $\delta_{+-}(t)$ (Fig. 1) and $\delta_{+-}$(Table I). In general, $\delta_{+-}$from MD is negative and $\left|\delta_{+-}\right|$is smaller than that from experiment. ${ }^{22,23}$ Despite the quantitative differences the values of $\left|\delta_{+-}\right|$from MD and experiment show a similar increase with $T$. It should be pointed out that uncertainties of $\delta_{+-}(t)$ and $\delta_{+-}$are important since they are not directly calculated or measured but determined as the difference between two very close quantities. Hansen and $\mathrm{McDonald}{ }^{8}$ observed from the experimental data for different molten alkali-metal salts ${ }^{23}$ that $\delta_{+-}$was negative in all cases. This finding was attributed to the formation of short-lived anion-cation complexes which do not contribute to electrical current. ${ }^{8}$ However, the values of $\delta_{+-}$cannot be only associated with the cross correlations between ions of opposite charge since, according to the results in this work, the effects of the correlations between distinct ions of the same species cannot be neglected [see Eqs. (6) and (9)].

It has been suggested that distinct properties may be used to determine the tendencies of the particles to diffuse together. ${ }^{27}$ The reasoning is as follows. If the coupling between the velocities of ions of different sign is stronger than that between ions of the same sign, it follows that 

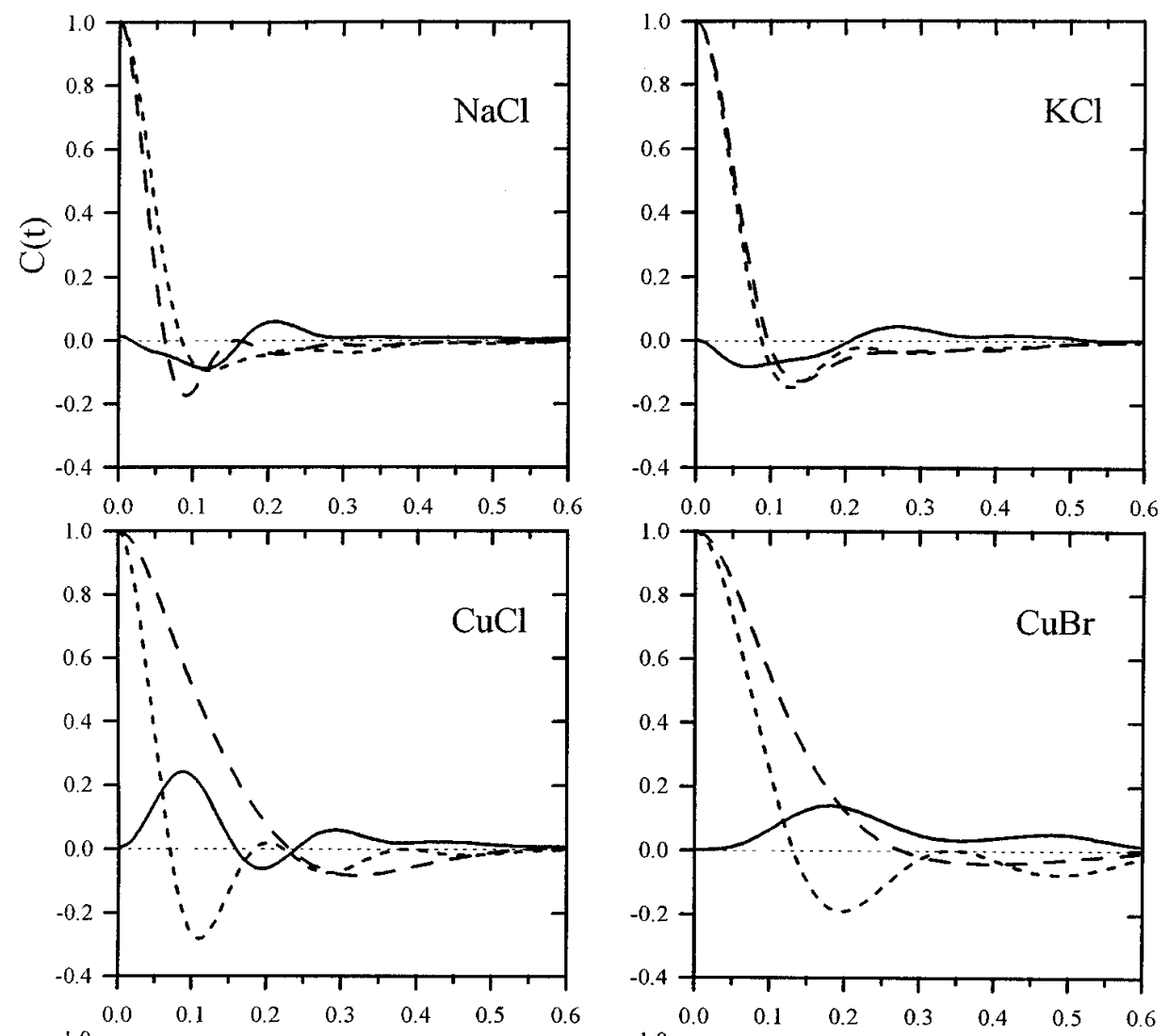

FIG. 1. Velocity autocorrelation functions and dynamic cross correlations $----C_{+}^{s}(t)$; ------$C_{-}(t) ;-\delta_{+-}(t)$.

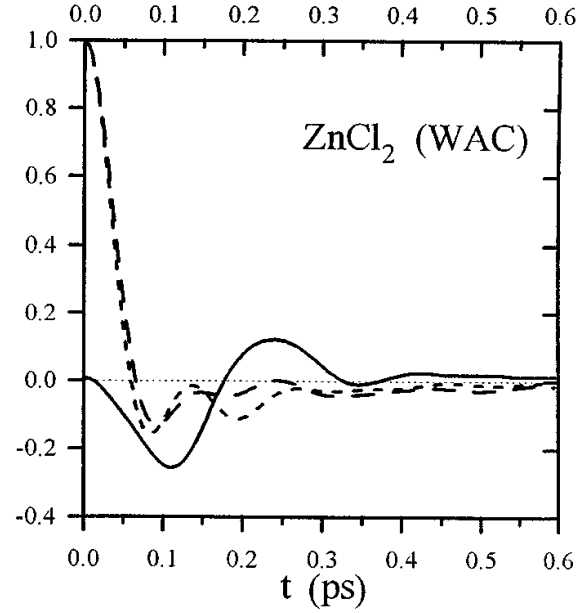

\section{Noble-metal halides: $\mathrm{CuCl}$ and $\mathrm{CuBr}$}

$D_{++}^{d R}+D_{--}^{d R}<2 D_{+-}^{d R}$ and, according to Eqs. (6) and (11), $D_{+-}^{d}<0$, and $\delta_{+-}<0$. However, we should be very cautious with this interpretation because, unlike $\Lambda_{+-}^{d}(t)$ and $D_{+-}^{d}$, the values of $\Lambda_{+-}^{d R}(t)$ and $D_{+-}^{d R}$ are strongly dependent on the considered reference frame. ${ }^{16}$ Moreover, the conclusions only based on the values of parameters such as $D_{+-}^{d}$ and $\delta_{+-}$may be misleading since, according to the results in Fig. 1 and those in Ref. 8, only the initial part of $\delta_{+-}(t)$ function tends to have negative values in systems as the molten alkali halides whereas the remaining part of $\delta_{+-}(t)$ may be positive. The minima of the $\delta_{+-}(t)$ functions are approximately reached at times close to the first zero of $C^{s}(t)$. This suggests that the anion-cation "associations", which may be associated with the Coulomb attraction are unstable since the lifetimes of these "pairs" ( $\cong 0.1 \mathrm{ps})$ are markedly lower than those estimated for the local structures in alkali halide melts $[\cong 2-3$ ps (Ref. 28)].
MD simulations of $\mathrm{CuCl}$ at three different temperatures $(T=773,973$, and $1073 \mathrm{~K})$ and $\mathrm{CuBr}(T=800 \mathrm{~K})$ have been carried out. As with alkali halides only the results at one temperature are explicitly reported in this paper. The effective pair potentials proposed by Stafford et al. ${ }^{3}$ have been assumed. This interaction model reproduces reasonably the experimental structure of copper halide melts. The potential form and parameters are detailed in Ref. 3. The potential for $\mathrm{CuCl}$ is shown in Fig. 1 of Ref. 17. Although the ionic sizes cannot be unambiguously defined, in the case of $\mathrm{CuCl}$ and $\mathrm{CuBr}$ anions are markedly bigger than cations and we can estimate that the ratio between their effective radii is about 4 for both systems. ${ }^{3}$

Although there are noticeable differences between the MD and experimental $D_{\alpha}^{s}$ results (Table I) the changes with $T$ [experimental data are only available for $\mathrm{CuCl}$ (Ref. 29)] 
TABLE I. Diffusion coefficients and electrical conductivities: Present MD results and experimental data.

\begin{tabular}{|c|c|c|c|c|c|c|c|c|}
\hline & & $\begin{array}{c}T \\
(\mathrm{~K})\end{array}$ & $\underset{\left(\text { ions } \AA^{-3} \text { ) }\right.}{\rho}$ & $D_{+}^{s}$ & $\begin{array}{r}D^{s} \\
10^{-5} \mathrm{c}\end{array}$ & $\left.\mathrm{n}^{2} \mathrm{~s}^{-1}\right)$ & $\left(\Omega^{-1} \mathrm{~cm}^{-1}\right)$ & $\delta+-$ \\
\hline $\mathrm{KCl}$ & 1173 & 0.0235 & $\begin{array}{l}7.8 \\
9.4\end{array}$ & $\begin{array}{l}7.8 \\
8.4\end{array}$ & 7.3 & $\begin{array}{l}2.7 \\
2.5 \\
2.5\end{array}$ & $\begin{array}{l}-0.7 \\
-0.25\end{array}$ & $\begin{array}{l}\text { MD } \\
\text { Expt. (Ref. 22) } \\
\text { Expt. (Ref. 23) }\end{array}$ \\
\hline $\mathrm{NaCl}$ & 1173 & 0.031 & $\begin{array}{r}8.6 \\
9.8 \\
11.4\end{array}$ & $\begin{array}{l}7.7 \\
7.8 \\
8.2\end{array}$ & 8.1 & $\begin{array}{l}4.0 \\
3.9 \\
3.9\end{array}$ & $\begin{array}{l}-0.0 \\
-0.11 \\
-0.20\end{array}$ & $\begin{array}{l}\text { MD } \\
\text { Expt. (Ref. 22) } \\
\text { Expt. (Ref. 23) }\end{array}$ \\
\hline $\mathrm{CuCl}$ & 773 & 0.0445 & $\begin{array}{l}9.3 \\
6.4\end{array}$ & $\begin{array}{l}2.4 \\
2.1\end{array}$ & 9.5 & $\begin{array}{l}10.0(2.5)^{\mathrm{a}} \\
3.7\end{array}$ & $\begin{array}{l}+0.6 \\
-0.2(+2.3)^{\mathrm{a}}\end{array}$ & $\begin{array}{l}\text { MD } \\
\text { Expt. (Ref. 29) }\end{array}$ \\
\hline $\mathrm{CuBr}$ & 800 & 0.0350 & 11.2 & 3.2 & 10.8 & $\begin{array}{l}8.8(2.0)^{\mathrm{a}} \\
2.6\end{array}$ & +0.5 & $\begin{array}{l}\text { MD } \\
\text { Expt. (Ref. 30) }\end{array}$ \\
\hline $\mathrm{SrCl}_{2}$ & 1260 & 0.0286 & $\begin{array}{l}0.8 \\
2.5\end{array}$ & $\begin{array}{l}2.1 \\
4.9\end{array}$ & 1.6 & $\begin{array}{l}1.4 \\
2.4\end{array}$ & +0.3 & $\begin{array}{l}\text { MD } \\
\text { Expt. }(\text { Ref. 36) } \\
\text { Expt. }(\text { Ref. 30) }\end{array}$ \\
\hline $\mathrm{ZnCl}_{2}$ & 1200 & 0.0296 & $\begin{array}{l}1.9 \\
0.7 \\
9.1\end{array}$ & $\begin{array}{r}2.8 \\
0.6 \\
14.7\end{array}$ & $\begin{array}{l}1.0 \\
0.5\end{array}$ & $\begin{array}{l}0.9 \\
0.4\end{array}$ & $\begin{array}{l}-0.5 \\
-0.3\end{array}$ & $\begin{array}{l}\mathrm{MD}^{\mathrm{d}} \\
\mathrm{MD}^{\mathrm{e}} \\
\text { Expt. (Ref. } 45)^{\mathrm{b}}\end{array}$ \\
\hline
\end{tabular}

${ }^{\text {a Values in parentheses are calculated with } z_{+} \text {and } z_{-} \text {corresponding to the effective charges in the potentials }}$ used in the MD simulations.

${ }^{\mathrm{b}}$ Extrapolated values. Densities are not given in Refs. 36 and 45 .

${ }^{c}$ Experimental value for a density of 0.0303 ions $\AA^{-3}$ (Ref. 30).

${ }^{\mathrm{d}} \mathrm{MD}$ results with the potential of Woodcock et al. (Ref. 38).

${ }^{\mathrm{e}} \mathrm{MD}$ results with the potential of Kumpta et al. (Ref. 44).

are reasonably reproduced by MD. As with alkali halides the accordance in the case of $D_{-}^{s}$ is quite good whereas larger disagreements are found for $D_{+}^{s}$. Nevertheless, the differences between MD and experiment are more important and in the opposite sense than for alkali halides. It is interesting to notice that $D_{+}^{s}$ is larger than $D_{-}^{s}$ in all cases, regardless of the relation between the mass of anions and cations. This corroborates earlier findings which indicate ${ }^{17}$ that the $D_{\alpha}^{s}$ coefficients in liquid noble-metal halides are mainly influenced by the ionic size differences. Electrical conductivities calculated from MD according to Eq. (14) with $\left|z_{+}\right|=\left|z_{-}\right|=1$ are much higher than the experimental data ${ }^{29,30}$ in all cases. This may be attributed to an incomplete ionization of these kind of molten salts. We have also calculated $\sigma$ using the same fractional charges as in the effective interionic potentials $(|z|=0.501$ for $\mathrm{CuCl}$ and $|z|=0.4828$ for $\mathrm{CuBr}$ (Ref. 3)]. Although the results are improved the new values of $\sigma$ are too low (Table I).

The $C_{\alpha}^{s}(t)$ functions for anions and cations show marked differences (Fig. 1). For both $\mathrm{CuCl}$ and $\mathrm{CuBr}$, independently of the relation between the ionic masses, $C_{-}^{s}(t)$ shows faster decay, more pronounced backscattering and more marked oscillations than $C_{+}^{s}(t)$. These findings reflect the existence of oscillatory motions of anions (the larger ions) into the cage made up by the neighboring ions of the same species, whereas the motions of cations are predominantly diffusive.
The $\delta_{+-}(t)$ and $\delta_{+-}$results (see Fig. 1 and Table I) reflect that the influence of the cross correlations for $\mathrm{CuCl}$ and $\mathrm{CuBr}$ is more important than for $\mathrm{KCl}$ and $\mathrm{NaCl}$. We emphasize the marked deviations from the Nernst-Einstein predictions revealed by the large values of $\delta_{+-}(\cong+0.5)$. Unlike for alkali halides, both the initial part of the $\delta_{+-}(t)$ functions and the $\delta_{+-}$values are positive. Hence, according to Eqs. (6), (11), and (17), $\left(D_{++}^{d R}+D_{--}^{d R}\right)$ is greater than $2 D_{+-}^{d R}$ which indicates that the coupling between the velocities of like ions is stronger than that between the ions of different sign. This finding suggests that the motion of anions is more affected by the collisions with other anions than by the attractive electrostatic interactions with cations. These results are consistent with earlier findings for soft-sphere mixtures ${ }^{16}$ which show that differences of size or mass between particles produce positive $\delta_{12}$ coefficients and $\delta_{12}(t)$ initial values.

Since the $\delta_{+-}$results from MD are determined through Eq. (17), they are independent of $|z|$. However, the experimental $\delta_{+-}$coefficients are determined from $\sigma, D_{+}^{s}$, and $D_{-}^{s}$ according to Eqs. (15) and (16) and their values depend on $|z|$. If $|z|=1$ the MD and experimental $\delta_{+-}$results for $\mathrm{CuCl}$ differ even in the sign. If the potential parameter $|z|$ is taken as an effective charge there are no differences in the sign of $\delta_{+-}$but the disagreements are still large (see Table I). These findings, as well as those for $\sigma$, are consistent with 
the assumption of an incomplete ionization but indicate that the $|z|$ parameter in the potential cannot be fully identified with an ionic effective charge.

\section{B. Dihalide melts: $\mathrm{Sr}_{2} \mathrm{Cl}$ and $\mathrm{Zn}_{2} \mathrm{Cl}$}

There are fewer computer simulation studies of 2:1 molten salts than of alkali halides. For $\mathrm{SrCl}_{2}, \mathrm{MC}$ (Ref. 31) and MD (Ref. 32) simulations as well as MHNC (modified hypernetted chain) calculations ${ }^{33}$ were carried out using an interionic potential of the Born-Mayer-Huggins form with the parameters derived by Busing. ${ }^{34}$ The resulting $g(r)$ functions reproduce the main features predicted by the neutronscattering data ${ }^{35}$ but significant disagreements are observed in the height of the first $g_{++}(r)$ and $g_{+-}(r)$ peaks. Moreover, the densities in these calculations were about $5 \%$ lower than the experimental values. We have performed a MD simulation at $1200 \mathrm{~K}$ and experimental density $(\rho$ $=0.0308$ ions $\AA^{-3}$ ) (Ref. 30) with the same potential model. The resulting properties do not show the characteristics of liquids but the trends typical of superionic systems, i.e., the cations form a solidlike sublattice and the anions have noticeable diffusion. We have also carried out a MD simulation of $\mathrm{SrCl}_{2}$ at $1260 \mathrm{~K}$ and the density obtained by Leeuw ${ }^{31}$ at this temperature (Table I). In this case, the resulting structure and diffusion of both anions and cations correspond to a melt. The results of this simulation, which are the ones discussed in this work, are consistent with findings in earlier studies. ${ }^{31-33}$ When compared with experiment, the structure is in qualitative agreement but the first $g_{++}(r)$ and $g_{+-}(r)$ peaks are too high. $D_{+}^{s}, D_{-}^{s}$, and $\sigma$ are of the same order of magnitude but markedly lower than the experimental data ${ }^{30,36,37}$ (Table I).

The resulting $C_{-}^{s}(t)$ for $\mathrm{SrCl}_{2}$ at $1260 \mathrm{~K}$ shows deeper backscattering and more pronounced oscillations than $C_{+}^{s}(t)$ (Fig. 1). The low values of the $D_{\alpha}^{s}$ coefficients from MD suggest that ionic oscillations are, probably, excessively enhanced. Moreover, the negative values of $\delta_{+_{-}}(t)$, which may be associated with anion-cation couplings, do not start until $t \cong 0.5 \mathrm{ps}$. This can be attributed to the cancellation of two opposite effects. On the one hand, the mass and size differences tend to produce positive $\delta_{+_{-}}(t)$ values. On the other hand, the electrostatic interactions tend to produce negative $\delta_{+-}(t)$ values. The value of $\delta_{+-}$from MD suggests that this parameter may be negative in liquid mixtures of highly charged ions. However, the experimental value of $\delta_{+-}$calculated from $D_{\alpha}^{s}$ in Ref. 36 and $\sigma$ in Ref. 30 is equal to -0.2 .

The former MD study of the $\mathrm{ZnCl}_{2}$ melt was carried out by Woodcock, Angell, and Cheeseman ${ }^{38}$ using a BornMayer-Huggins-type interionic potential (WAC potential). Later, several potential models were proposed ${ }^{39-42}$ but none of them can reproduce correctly the $\mathrm{ZnCl}_{2}$ structure, particularly the first peak of $g_{++}(r) .{ }^{43}$ Kumta, Deymeir, and Risbud $^{44}$ developed an interaction potential (KDR potential). The $g(r)$ 's obtained by MD at constant $T(1200 \mathrm{~K})$ and $P(1$ atm) using the KDR model show good agreement with the experimental data at $T=600 \mathrm{~K}$ but the resulting density was about $40 \%$ lower than the experimental value at $T$ $=1200 \mathrm{~K}$. We have simulated $\mathrm{ZnCl}_{2}$ at $1200 \mathrm{~K}$ and experimental density (Table I) using both the WAC and the KDR

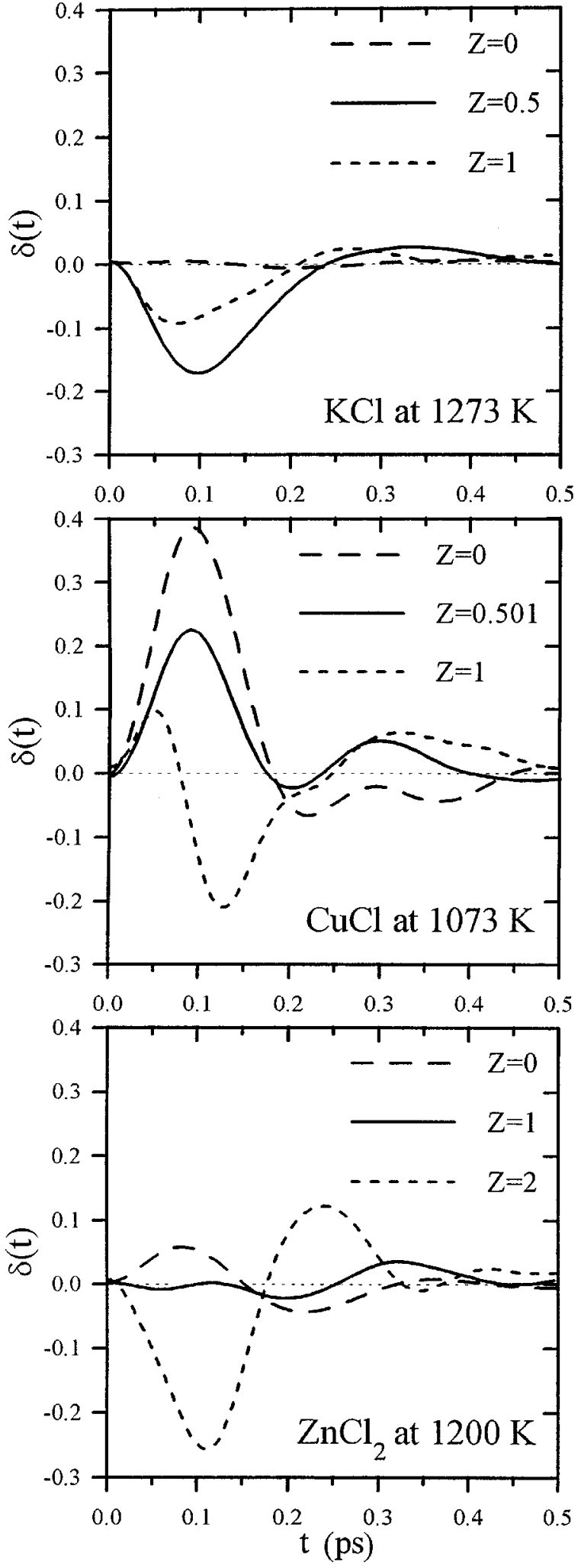

FIG. 2. Dynamic cross correlations $\left[\delta_{+-}(t)\right]$ for systems with different ionic charges.

potentials. The $g_{--}(r)$ and $g_{++}(r)$ functions resulting from neutron-scattering measurements at $600 \mathrm{~K}$ (Ref. 43) show peaks of very similar heights $\left[g\left(r_{\max } \cong 3\right)\right]$ located at very close positions ( $r_{\max }=3.7-3.8 \AA$ ). This is in disagreement with the WAC results but the experimental positions of the 
TABLE II. Diffusion coefficients of $\mathrm{KCl}-, \mathrm{CuCl}-$, and $\mathrm{ZnCl}_{2}$-like systems with different ionic charges.

\begin{tabular}{|c|c|c|c|c|c|c|c|}
\hline & $\begin{array}{c}T \\
(\mathrm{~K})\end{array}$ & $\begin{array}{c}\rho \\
\left(\text { ions } \AA^{-3} \text { ) }\right.\end{array}$ & $z$ & $D_{+}$ & $\begin{array}{c}D_{-} \\
\left(10^{-5} \mathrm{~cm}^{2} \mathrm{~s}^{-1}\right)\end{array}$ & $D_{+-}$ & $\delta_{+-}$ \\
\hline & & & 0 & 25.2 & 23.0 & 25.0 & 0.0 \\
\hline \multirow[t]{3}{*}{$\mathrm{KCl}$} & 1273 & 0.023 & 0.5 & 17.3 & 16.6 & 11.3 & -0.3 \\
\hline & & & 1 & 10.2 & 10.1 & 9.0 & -0.12 \\
\hline & & & 0 & 23.4 & 5.4 & 20.0 & +0.4 \\
\hline \multirow[t]{3}{*}{$\mathrm{CuCl}$} & 1073 & 0.0415 & 0.501 & 16.5 & 6.3 & 16.5 & +0.45 \\
\hline & & & 1 & 4.1 & 2.7 & 3.0 & -0.1 \\
\hline & & & 0 & 16.8 & 15.0 & 16.2 & 0.0 \\
\hline \multirow[t]{2}{*}{$\mathrm{ZnCl}_{2}{ }^{\mathrm{a}}$} & 1200 & 0.0296 & 1 & 5.5 & 10.1 & 8.2 & +0.2 \\
\hline & & & 2 & 1.9 & 2.8 & 1.0 & -0.5 \\
\hline
\end{tabular}

${ }^{a}$ MD simulations using the WAC (Ref. 38) potential.

peaks are well reproduced by the KDR potential. The MD diffusion coefficients are markedly lower than the ones obtained by extrapolating to $1200 \mathrm{~K}$ the experimental data between 600 and $800 \mathrm{~K}^{45}$ The disagreement is more important in the case of the KDR potential (Table I). These findings suggest that the structure is more realistically reproduced by the KDR model whereas dynamic properties are better reproduced by the WAC model.

The $C^{s}(t)$ and $\delta_{+_{-}}(t)$ functions obtained with the WAC model are shown in Fig. 1. $C_{-}^{s}(t)$, which corresponds to the lighter particles, is more oscillatory than $C_{+}^{s}(t)$. Moreover, $\delta_{+-}(t)$ shows a negative initial region which may be associated with the existence of strong anion-cation couplings. The $C^{s}(t)$ and $\delta_{+-}(t)$ results using the KDR model show similar qualitative features but marked quantitative differences with respect to those using the WAC model. Unlike for $\mathrm{SrCl}_{2}$, the MD values of $\delta_{+-}$for $\mathrm{ZnCl}_{2}$ are positive (Table I).

\section{Influence of the ionic charge: $\mathrm{KCl}-$, $\mathrm{CuCl}-$, and $\mathrm{ZnCl}_{2}$-like systems}

The results discussed in the preceding paragraphs indicate that the values of $\delta_{+-}(t)$ and $\delta_{+-}$are the result of several contributions including the electrostatic interactions and the ionic size and mass differences. In order to analyze the influence of the electrostatic interactions on the dynamic distinct correlations, we have performed MD simulations of several systems with identical particles except their ionic charges $\left(\left|z_{+}\right| \equiv z\right)$. The effects of the mass and size differences were already discussed in Ref. 16.

$\mathrm{KCl}$ at $1273 \mathrm{~K}, \mathrm{CuCl}$ at $1073 \mathrm{~K}$, and $\mathrm{ZnCl}_{2}$ at $1200 \mathrm{~K}$ (assuming the WAC potential) have been considered. MD simulations of KCl-like systems with $z=0$ and $z=0.5$, $\mathrm{CuCl}$-like systems with $z=0$ and $z=1$, and $\mathrm{ZnCl}_{2}$-like systems with $z=0$ and $z=1$ have been carried out. These simulations correspond to neutral and partially ionized systems in the case of $\mathrm{KCl}$ and $\mathrm{ZnCl}_{2}$ and to neutral and fully ionized systems in the case $\mathrm{CuCl}$ (a partial charge, $z=0.501$, was attributed to the actual system). The results for ideal and actual systems are compared in Fig. 2 and Table II. The methodology used in this study is analogous to that in earlier analyses of the effects of the ionic charge on the structure and single dynamical properties of $\mathrm{NaCl}$ and $\mathrm{CuCl}^{17} \mathrm{~A}$ correct interpretation of the results in this section requires us to consider that changes in the ionic charges modify the effective ionic sizes and produce important changes in the structure of the systems. ${ }^{17}$

The distinct time correlation functions for systems with different ionic charges corroborate the conclusions in Secs. III A and III B. When $z$ increases, the influence of the electrostatic interactions becomes more important and the initial values of $\delta_{+_{-}}(t)$ tend to be more negative (Fig. 2). However, $\delta_{+-}(t)$ for $\mathrm{KCl}$ with $z=0.5$ shows a minimum deeper than with $z=1$ which may be related to the less ordered structure of the former system. For $\mathrm{CuCl}$ the influence of the size and mass differences between anions and cations is predominant and the $\delta_{+-}(t)$ functions only show large negative values for $z=1$. The results for $\mathrm{ZnCl}_{2}$ are very illustrative, i.e., the initial $\delta_{+-}(t)$ values for $z=0$ reflect the influence of the size and mass differences whereas for $z=2$ the influence of the electrostatic interactions is dominant and for $z=1$ the two competing influences are of similar importance. The values of the $\delta_{+-}$coefficients cannot be easily interpreted because they correspond to the time integrals of $\delta_{+-}(t)$ functions with marked oscillatory shapes.

\section{CONCLUDING REMARKS}

$D_{\alpha}^{s}$ and $\sigma$ coefficients resulting from MD simulations with the usual pairwise interionic potentials have been compared with the available experimental data. For molten alkali halides, the calculated $D_{+}^{s}$ coefficients are somewhat lower than the experimental ones, whereas $D_{-}^{s}$ and $\sigma$ are close to the experimental findings. For $\mathrm{CuCl}$ the discrepancies are larger but the MD results reproduce qualitatively the differences between the $D_{\alpha}^{s}$ coefficients for anions and cations. The $\sigma$ results for noble-metal halides suggest that the parameter $|z|$ in the potential cannot be fully associated with an effective ionic charge. In the case of dihalide melts the disagreements between MD and experiment are large for both structural and dynamical properties. Recent findings suggest that the consideration of dipole and quadrupole polarization in the potential can improve significantly the results and can be essential in the case of the $2-1$ systems. ${ }^{46}$

The dynamic behavior of ions has been analyzed through $C_{+}^{s}(t), C_{-}^{s}(t)$, and $\delta_{+_{-}}(t)$. In molten alkali halides, $C_{\alpha}^{s}(t)$ for the lighter ions shows a faster initial decay, more pronounced backscattering, and more marked oscillations than for the heavier ions. Moreover, the $\delta_{+_{-}}(t)$ functions 
show positive initial values which may be associated with couplings among the velocities of ions with opposite sign. The results for molten noble-metal halides are very different. $C_{\alpha}^{s}(t)$ for the larger ions shows a faster decay, deeper backscattering, and more marked oscillations than for the smaller ions and the ionic mass plays a minor role. Moreover, the $\delta_{+-}(t)$ functions show negative initial values which indicate that the couplings among the velocities of ions with the same charge are stronger than those among ions of opposite sign. In these systems the dynamic correlations are very much influenced by the collisions between big ions which hinder considerably their motions. ${ }^{17}$ The results for systems with different ionic charges have corroborated that the dynamic cross correlations in molten salts are the result of two opposite tendencies, namely the electrostatic interactions and the mass and/or size differences between anions and cations. These competing tendencies are also reflected by the results for dihalide melts which are closer to those for alkali halides as correspond to systems of particles with strong Coulomb interactions.

Significant deviations from the Nernst-Einstein relation have been found for noble-metal halides. Our findings suggest that, although $\delta_{+-}$was negative for simple molten salts and different alkali metals salts, ${ }^{26}$ this fact cannot be taken as a general rule. Finally, we want to emphasize that the values of both $\delta_{+-}$and the $\delta_{+-}(t)$ function after its first minimum are the result of complex processes involving several particles. Accordingly, we should be very cautious with the analysis of the experimental conductivities and distinct diffusion coefficients, and we cast doubt on the conclusions about association of particles in liquid mixtures which are simply based on the values of the $\delta_{+-}$coefficients. ${ }^{11,27}$

\section{ACKNOWLEDGMENTS}

This work has been supported by DGICYT through Grant No. PB93-0971-C03 and by CIRIT through a SGR grant.
${ }^{1}$ M. J. L. Sangster and M. Dixon, Adv. Phys. 25, 247 (1976).

${ }^{2}$ L. V. Woodcock and K. Singer, Trans. Faraday Soc. 67, 12 (1971).

${ }^{3}$ A. J. Stafford, M. Silbert, J. Trullàs, and A. Giró, J. Phys. Condens. Matter 2, 6631 (1990).

${ }^{4}$ M. Rovere and M. P. Tosi, Rep. Prog. Phys. 49, 1001 (1986).

${ }^{5}$ R. L. McGreevy and L. Pusztai, Proc. R. Soc. London Ser. A 430, 241 (1990).

${ }^{6}$ M. P. Tosi, D. L. Price, and M. L. Saboungi, Annu. Rev. Phys. Chem. 44, 173 (1993).

${ }^{7}$ J. P. Hansen and I. R. McDonald, J. Phys. C. 7, L384 (1974).

${ }^{8}$ J. P. Hansen and I. R. McDonald, Phys. Rev. A 11, 2111 (1975).

${ }^{9}$ G. Ciccotti, G. Jacucci, and I. R. McDonald, Phys. Rev. A 13, 426 (1976).

${ }^{10}$ H. L. Friedman, F. O. Rainieri, and M. D. Wood, Chem. Scr. 29A, 49 (1989), and references therein.

${ }^{11}$ R. Mills, R. Malhotra, L. A. Woolf, and D. G. Miller, J. Phys. Chem. 98, 5565 (1994).

${ }^{12}$ J. A. Padró, J. Trullàs, and G. Sesé, Mol. Phys. 72, 1035 (1991).

${ }^{13}$ J. Trullàs and J. A. Padró, in Molecular Liquids: New Perspectives in Physics and Chemistry, edited by J. J. C. Teixeira-Dias (Kluwer, Dordrecht, 1992), p. 393.

${ }^{14}$ F. O. Rainieri and H. L. Friedman, J. Chem. Phys. 91, 5633 (1989).

${ }^{15}$ J. Trullàs and J. A. Padró, J. Chem. Phys. 99, 3983 (1993).

${ }^{16}$ J. Trullàs and J. A. Padró, Phys. Rev. E 50, 1162 (1994).

${ }^{17}$ J. Trullàs, A. Giró, J. A. Padró, and M. Silbert, Physica A 171, 384 (1991).

${ }^{18}$ J. Trullàs, A. Giró, and M. Silbert, J. Phys. Condens. Matter 2, 6643 (1990).

${ }^{19}$ F. G. Fumi and M. P. Tosi, J. Phys. Chem. Solids 25, 31 (1964).

${ }^{20}$ P. Ballone, G. Pastore, and M. P. Tosi, J. Chem. Phys. 81, 3174 (1984).

${ }^{21}$ M. Wilson and P. A. Madden, J. Phys. Condens. Matter 5, 2687 (1993).

${ }^{22}$ G. J. Janz, C. B. Allen, N. P. Bansal, R. M. Murphy, and R. P. T. Tomkins, Molten Salts: Vol. 2, NSRDS Natl. Bur. Stand. (U.S.) No. 61 (U.S. GPO, Washington, DC, 1979).
${ }^{23}$ R. E. Young and J. P. O'Connell, Ind. Eng. Chem. Fundam. 10, 418 (1971)

${ }^{24}$ G. Jacucci, I. R. McDonald, and A. Rahman, Phys. Rev. A 13, 1581 (1976).

${ }^{25}$ M. Balcells, A. Giró, and J. A. Padró, Physica 135 A, 414 (1986).

${ }^{26}$ J. P. Hansen and I. R. McDonald, Theory of Simple Liquids (Academic, London, 1986), Chap. 10.

${ }^{27}$ T. Kato, J. Phys. Chem. 89, 5750 (1985).

${ }^{28}$ F. Lantelme and P. Turq, J. Chem. Phys. 81, 5046 (1984).

${ }^{29}$ J. C. Poignet and M. J. Barbier, Electrochim. Acta 26, 1429 (1981).

${ }^{30}$ G. J. Janz, F. W. Dampier, P. K. Lakshminarayanan, P. K. Lorenz, and R. P. T. Tomkins, Moten Salts: Vol. 1, NSRDS Natl. Bur. Stand. (U.S.) No. 15 (U.S. GPO, Washington, DC, 1968).

${ }^{31}$ S. Leeuw, Mol. Phys. 36, 103 (1978).

${ }^{32}$ S. Leeuw, Mol. Phys. 36, 765 (1978).

${ }^{33}$ G. Pastore, P. Ballone, and M. P. Tosi, J. Phys. C 19, 487 (1985).

${ }^{34}$ R. W. Busing, Trans. Am. Crystallogr. Assoc. 6, 57 (1970).

${ }^{35}$ R. L. McGreevy and E. W. J. Mitchell, J. Phys. C 15, 5537 (1982).

${ }^{36}$ J. O’M. Bockris, S. R. Richards, and L. Nanis, J. Phys. Chem. 69, 1627 (1965)

${ }^{37}$ M. Wilson and P. A. Madden, J. Phys. Condens. Matter 5, 6833 (1993).

${ }^{38}$ L. V. Woodcock, C. A. Angell, and J. Cheeseman, J. Chem. Phys. 65, 1565 (1976).

${ }^{39}$ P. J. Gardner and D. M. Heyes, Physica B 131, 227 (1985).

${ }^{40}$ P. Ballone, G. Pastore, J. S. Thakur, and M. P. Tosi, Physica B 142, 294 (1986).

${ }^{41}$ S. Inoue, M. Tamaki, H. Kawazoe, and M. Yamane, J. Mater. Res. 2, 357 (1987).

${ }^{42}$ K. Hirao and N. Soga, J. Non-Cryst. Solids 95-96, 577 (1987).

${ }^{43}$ S. Biggin and J. E. Enderby, J. Phys. C 14, 3129 (1981).

${ }^{44}$ P. N. Kumta, P. A. Deymier, and S. H. Risbud, Physica B 153, 85 (1988).

${ }^{45}$ C. A. Sjöblom and A. Behn, Z. Naturforsch. Teil A 23, 495 (1968).

${ }^{46}$ M. Wilson, P. A. Madden, and B. J. Costa-Cabral, J. Phys. Chem. 100, 1227 (1996). 